\title{
Expedient Method for Direct Mono-amidation of Phosphonic and Phosphoric Acids
}

\author{
Victoria C. Yan ${ }^{*}$, Cong-Dat Pham 1 , and Florian L. Muller1
}

1Department of Cancer Systems Imaging, University of Texas MD Anderson Cancer

Center, Houston, TX, 77054, USA

${ }^{*}$ Correspondence should be addressed to Victoria C. Yan, Department of Cancer

Systems Imaging, University of Texas MD Anderson Cancer Center, Houston, TX

77054. E-mail: victoriacyanide@gmail.com

Previous work:

a.) Atherton-Todd reaction

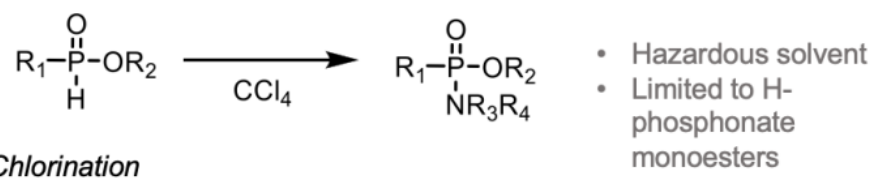

b.) Chlorination

monoesters

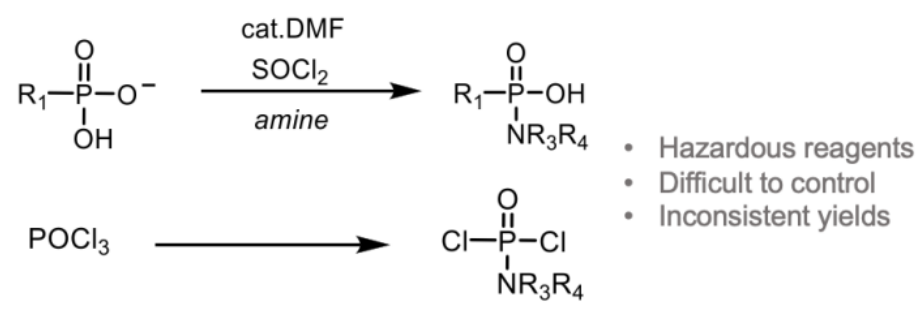

c.) Peptide coupling

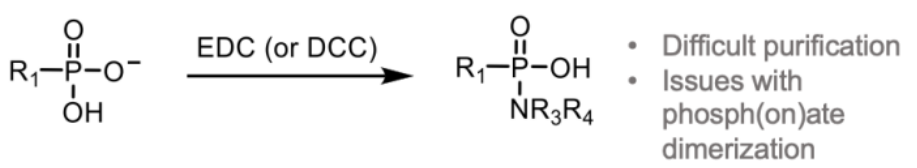

This work:

c.) Novel Mitsunobu Coupling

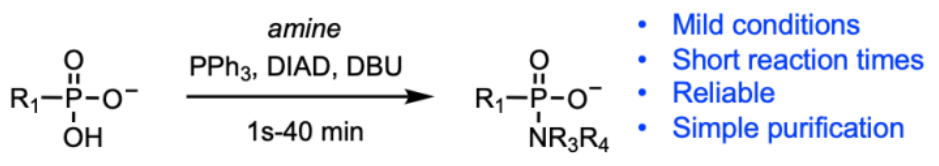

$\mathrm{R}_{1}, \mathrm{R}_{2}=$ O-ester, aryl, benzyl $\mathrm{R}_{3}=\mathrm{H}, \mathrm{Me}, \mathrm{R}_{4}=$ benzyl, aliphatic 
ABSTRACT. Mono-amidated $\mathrm{P}(\mathrm{V})$ pro-drugs predominately contribute to the vastly improved delivery of phosphate and phosphonate-containing anti-viral/cancer nucleotide analogues. However, synthetic approaches towards their formation are often harsh and unreliable, which may hamper the identification of novel, more effective amine pro-drugs. Here, we show that direct mono-amidation of structurally complex phosphonic and phosphoric acids may be accomplished in as quickly as seconds by re-purposing the $\mathrm{PPh}_{3} / \mathrm{DIAD}$ redox pair. Where the triphenylphosphine oxide byproduct is often cited as a vulnerability, we use its formation as an asset. Juxtaposing the anionic nature of the generated mono-amidated product, the desired product may be isolated with a single water extraction. Compared to state-of-the-art strategies towards phosphoramidates, our approach is mild, reliable, and enables access to a variety of aliphatic and benzylic amines for pro-drug attachment.

INTRODUCTION. Phosphoramidates and phosphonoamidates are characterized by a single $\mathrm{P}(\mathrm{V})-\mathrm{N}$ bond and are chemical moieties with high therapeutic relevance $[1,2]$. From Tenofovir Alafenamide[3], to Sofosbuvir[4], to Remdesivir[5], the investigational drug for treating COVID-19 (NCT04280705), phosphoramidates permeate the antiviral pro-drug landscape. Yet for all their importance in improving the delivery of phosph(on)ate drugs, current mono-amidation strategies are subverted by their harsh reaction conditions, challenging purifications, and inconsistent yields[6]. Two examples of common monoamidation approaches include chlorination and peptide-like couplings. The former method not only requires the use of hazardous reagents, but also demands great precaution for handling the resulting phosphoryl dichloride, which is highly susceptible to hydrolysis. The 
latter approach is challenged by the propensity for phosphonates and phosphates to dimerize, requiring vast excesses of amine and coupling reagent to obtain the monoamidated product[7]. Here, we show that direct mono-amidation of phosphonic or phosphoric acids may be accomplished using $\mathrm{PPh}_{3}$ and diisopropyl azodicarboxylate (DIAD) to expediently generate structurally diverse phosphonoamidates and phosphoramidates.

RESULTS AND DISCUSSION. We first discovered the reaction while synthesizing mixed phosphoramidate pro-drugs of our laboratory's phosphonate-containing Enolase inhibitor, hydrogen (1-hydroxy-2-oxopiperidin-3-yl)phosphonic acid (HEX)[8]. We first combined $\mathrm{PPh}_{3}$ (2 equiv.) and DIAD (2 equiv.) in dichloromethane (DCM) at $0^{\circ} \mathrm{C}$ for 30 minutes to form the betaine[9]. Thereafter, we prepared a solution of a benzyl-protected precursor to HEX, BnHEX (1 equiv.), and amine (2 equiv.) in DCM, which was then added dropwise to the betaine solution. After 30 minutes of reaction, we found that the desired mono-adduct was efficiently formed, as indicated by a singlet at $\sim 18 \mathrm{ppm}$ by ${ }_{31} \mathrm{P}$ NMR, with minimal phosphonate dimerization ( 10 ppm). Our initial results show preferential reaction with benzylic amines, with the most effective reaction being with 2-picolylamine (Figure 1a, entry 2). Having established the general reaction parameters for phosphonic acids, we sought to examine the reaction efficiency when using phosphates as pro-nucleophiles. Using 1-naphtyl phosphate as a test compound, we screened a variety of aromatic and aliphatic amines (Figure 1b, entries 1-11). Compared to our reactions with BnHEX, we found that pre-formation of the betaine solution was not necessary to prevent phosphate dimerization. Sequential addition of the phosphate, amine, PPh3, and DIAD in NMP at 
room temperature was all that was required for the desired phosphoramidate to be generated instantaneously (Figure 3a). Optimization for the phosphoric acid reactions showed that the acid, amine, phosphine, and azo compound could be added in a 1:2:1:1 ratio, which was lower than the 2 equivalents of phosphine and azo compound required for efficient phosphonoamidate formation. This can likely be attributed to the significantly faster rate of the reactions with phosphonic acids, compared to phosphonic acids (Figure 3a). We then applied our reaction directly to Fludarabine monophosphate (Figure 1c, entries 12-15). Inherent in this decision was our desire to test the limits of our reaction's exclusivity for phosphates. The heterocycle component of Fludarabine monophosphate is analogous to that of adenine and contains the acidic N1-nitrogen (pKa $\sim 4.2$ ) and the C6-amine (pKa 9.8). Fludarabine monophosphate also has two potentially reactive hydroxyls in the ribose sugar. Yet even with these vulnerabilities, which often demand protecting groups[10], coupling with an amine solely occurred with the phosphate (Supplementary Figure S3).

Between our reactions with phosphonic acid BnHEX and phosphates, we found that structurally diverse amines were much more amenable to being attached onto the latter. Structurally, the difference between phosphonic- and phosphoric acids is subtle. However, this dissimilarity accounts for a distinct difference in pKas in the acidic hydroxyl groups. While the predicted pKas of the first acidic hydroxyl group in both species is $\sim 1.8$, the pKa of the second acidic hydroxyl group for phosphoric acids is $\sim 6.8$, compared to $\sim 8.3$ for phosphonic acids. We thus reasoned that the second hydroxyl group in phosphoric acids was likely deprotonated under the mildly alkaline conditions of the 
reaction; this was unlikely to hold true for phosphonic acids. Though the mechanistic rationale behind the significance of this difference remains unclear to us, we hypothesized that deprotonation of the second hydroxyl group in phosphonic acids could enable efficient attachment of aliphatic amines in a manner similar to that observed for phosphoric acids. Returning to our reaction setup for BnHEX, we added the strong, nonnucleophilic base 1,8-diazabicyclo[5.4.0]undec-7-ene (DBU, 2 equiv.) to our solution containing BnHEX and amine, which was then added dropwise to the betaine solution. Indeed, we found that this allowed attachment of previously inaccessible aliphatic amines to $\mathrm{BnHEX}$ at remarkable yields (Figure $\mathbf{1 b}$, yield 2). It is reasonable to conclude that the addition of DBU to any of the amine couplings described will greatly improve reaction yields.

Seeking to test the limits of our reaction even further, we chose to apply these coupling conditions to other therapeutically relevant molecules such as Adefovir and Tenofovir monophosphonate. From our reactions with BnHEX, we had already learned that phosphonic acids were poorly suited to coupling with amines in highly polar solvents such as NMP and DMF (Supplementary Table S1)-preferring non-polar solvent such as $\mathrm{DCM}$ and $\mathrm{CHCl}_{3}$. Indeed, pilot reactions in NMP with these phosphonic acids and the most amenable amine, 2-picolylamine, proved unfruitful. However, with our discovery that DBU can facilitate the couplings between BnHEX and aliphatic amines, we proceeded to conduct with our reactions with Adefovir and Tenofovir using the same procedure as we had done for BnHEX. To our surprise, the addition of 2 equivalents of DBU was able to dissolve these phosphonic acids in $\mathrm{DCM}$ and $\mathrm{CHCl}_{3}$ and the coupling product with 2- 
picolylamine occurred in moderate yields (Figure 1c). Extending this logic, we then decided to test whether HEX, the de-benzylated active Enolase inhibitor, would be able to undergo amine coupling using the DBU/DCM procedure. Much like Adefovir and Tenofovir, HEX was only soluble in highly polar organic solvents, precluding the reaction prior to discovering the effects of DBU. Indeed, we found that even HEX, with its reactive hydroxamic acid could be coupled with 2-picolylamine with the addition of 2 equivalents of DBU (Figure 1c). The stark contrast in reactivity for this panel of phosphonic acids supports the finding that amine coupling under these conditions with phosphonates must occur in non-polar solvents. To test the unlikely possibility that DBU, rather than solvent choice, was the predominant reason for the reaction's improved efficacy, we also performed the coupling between BnHEX and 2-picolylamine using 2 equivalents of DBU in NMP. After forming the betaine and reacting with the BnHEX/2-picolylamine/DBU mixture for 40 minutes, there was no reaction (Supplementary Table S1). These data further highlight the necessity for phosphonate-amine couplings to occur in non-polar solvents.

Prompted by our finding that the dissolution of and reaction with phosphonic acids in nonpolar solvents can be facilitated by addition of DBU, we sought to examine whether similar observations could be made for phosphonic acids. Based on conventional Mitsunobu couplings, we anticipated that the mono- and dibasic phosphates would not readily couple under these conditions, as an acidic proton is necessary for protonation of the betaine in the opening steps of the reaction mechanism[11]. Indeed, we found that, even with the addition of DBU (0.75-2 equivalents), mono- and dibasic phosphates were essentially 
insoluble in $\mathrm{DCM}$ and $\mathrm{CHCl}_{3}$. However, for fully protonated phosphoric acids, such as 1naphthyl phosphate (dihydrogen, Figure $1 \mathrm{~b}$ ), 0.75 equivalents of DBU was the minimum amount required for dissolution in either $\mathrm{DCM}$ or $\mathrm{CHCl}_{3}$ after light vortexing. Overall, reaction with various benzylic and aliphatic amines proved more effective in these nonpolar solvents compared to those performed in NMP without DBU (Figure 1b, yield 1 versus yield 2). This suggests that, even for phosphonic acids, mono-amidation likely occurs more readily occurs in non-polar solvents compared to highly polar ones. However, the acidity of phosphoric acids still enables the reaction to occur quite efficiently in solvents such as NMP. We note that 1-naphtyl phosphate served as a very user-friendly compound to make these observations due to its ability to dissolve in the aforementioned solvent systems. In contrast, fludarabine monophosphate proved essentially insoluble in the DBU/DCM despite being a dihydrogen species. Here, comparison of reaction efficiencies in different solvent systems was not possible (Figure 1b). Thus, to ensure that these solvent-specific effects were not unique to 1-naphtyl phosphate, we also coupled phenyl dihydrogen phosphate with benzylamine in either NMP or DBU/DCM. Consistent with our observations with 1-naphthyl phosphate, we found that reaction efficiency was improved in the latter, non-polar solvent system (Figure 1c).

Finally, we sought to gain mechanistic insight into our reaction. While the $\mathrm{PPh}_{3} / \mathrm{DIAD}$ redox pair is synonymous with Mitsunobu couplings, examination of the classical Mitsunobu reaction mechanism[11] proves to be incompatible with our transformation. In canonical Mitsunobu couplings with alcohols, oxidation of $\mathrm{PPh}_{3}$ to triphenylphosphine oxide (TPPO) occurs due to formation of the oxygen-PPh3 complex, followed by an Sn2 
attack on the alcoholic carbon-resulting in the oxygen transfer from the alcohol to the phosphine to form the oxide. In our case, there is no such alcohol oxygen to perform this transfer to generate TPPO. We thus propose an amidation mechanism wherein the phosphonic or phosphoric acid acts as the pro-electrophile, rather than its traditional role as the pro-nucleophile (Figure 3b). Within our model, oxidation of the phosphine occurs through oxygen transfer from the phosphoric or phosphonic acid, as no other likely oxygen donors are present amongst the starting reagents. To gain insight into our proposed reaction mechanism, we prepared bis-18O-labeled 1-naphthyl phosphate, which was then HPLC-purified to remove any trace of residual $\mathrm{H}_{218} \mathrm{O}$. The labeled phosphate was then reacted it with S)-(-)-a-methylbenzylamine (Figure 3c). Compared to the control, unlabeled reaction, we found a significantly greater proportion of $18 \mathrm{O}$-labeled TPPO (Figure 3c, $M+1=281.29$ expected, 281.27 found), which coincided with the majority of the mono-18O-labeled phosphoramidate product (Figure $3 \mathbf{c}, \mathrm{M}+1=330.32$ expected, 330.34 found). These observations strongly support our proposed mechanism of oxygen transfer to the phosphine, thereby strengthening the role of the phosphoric or phosphonic acid as the pro-electrophile in our mono-amidation reactions.

In sum, we have discovered a novel variation on the Mitsunobu coupling that enables expedient, direct mono-amidation of highly functionalized phosphate or phosphonatecontaining species. In contrast to other coupling procedures, our reaction is fast, reliable, and occurs under exceptionally mild conditions. At the fundamental level, our reaction turns the formation of TPPO-a historically perceived inconvenience-into an asset: 1 water extraction is all that is required for its removal and to obtain the mono-amidated 
product at sufficient purity to proceed with the next reaction. That our reaction is exquisitely selective for phosphates or phosphonates enables direct reaction onto highly functionalized nucleotide analogues for pro-drugging efforts. The burgeoning applications of the ProTide pro-drug strategy[12] amidst the interest in discovering novel phosphoramidate-based pro-drugs make our reaction well-suited to rapidly screening a number of biologically-labile amines. Taken together, our reaction not only expands the reaction scope of the traditional Mitsunobu coupling[13] but also represents a significant addition to the phosph(on)ate chemist's toolkit.

AUTHOR CONTRIBUTIONS. V.C.Y. conceived of the synthesis and designed experiments. V.C.Y. and C.D.P. performed syntheses and characterized compounds. V.C.Y. wrote the manuscript. F.L.M. provided critical comments and oversaw the project. All authors have given approval to the final version of the manuscript.

ACKNOWLEDGEMENTS. We thank Gary J. Snyder and Darren G. Hamilton for critical comments and suggestions and Kumar Kalurachchi for NMR assistance. This work is supported by the American Cancer Society (RSG-15-145-01-CDD) and the National Comprehensive Cancer Network (YIA170032).

AUTHOR DISCLOSURES. V.C.Y., C.D.P., and F.L.M. are inventors on a patent application describing a novel method for the mono-amidation of phosphates and phosphonates. 
A

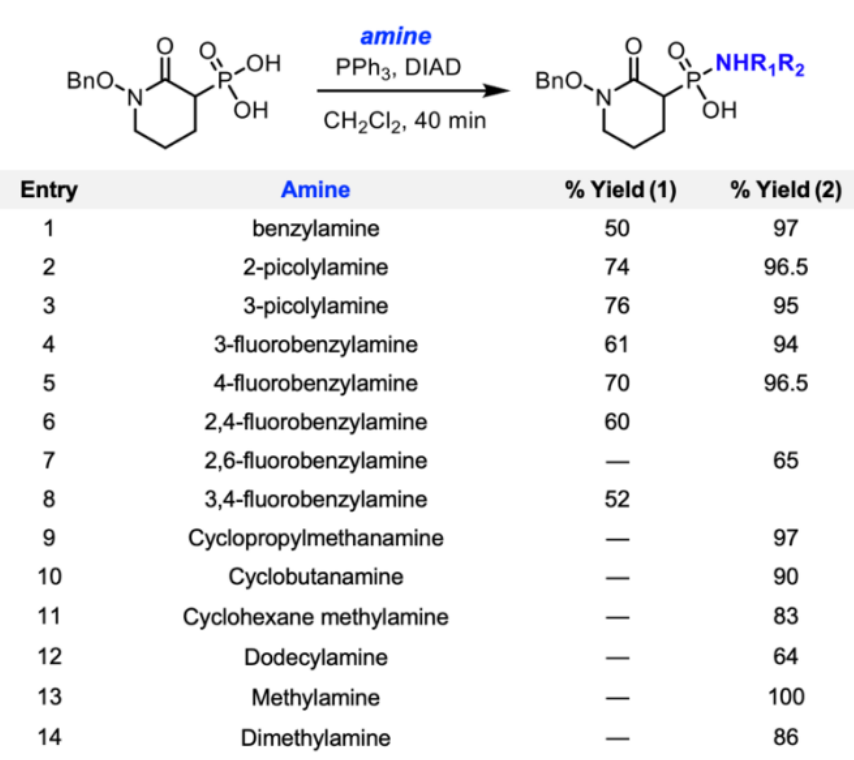

C Therapeutically relevant $\mathrm{P}(\mathrm{V})$ molecules
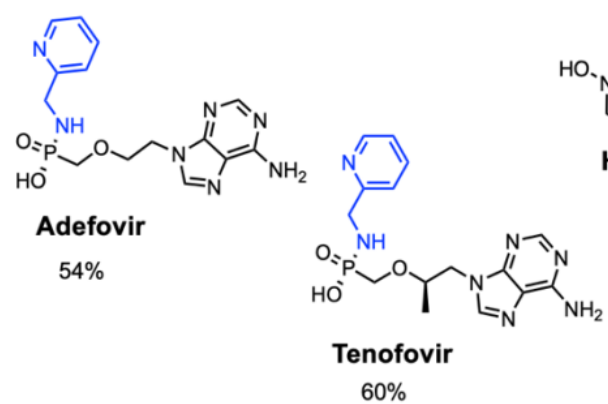

B

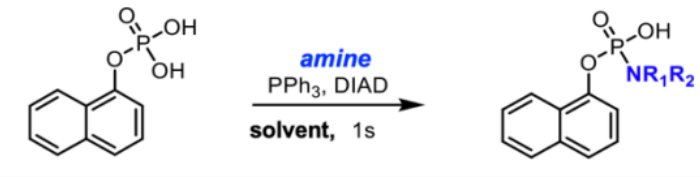

$\begin{array}{cccc}\text { Entry } & \text { Amine } & \text { \% Yield (1) } & \text { \% Yield (2) } \\ 1 & \text { benzylamine } & 70 & 100\end{array}$

$\begin{array}{llll}2 & \text { 2-picolylamine } & 76 & 87.5\end{array}$

3 4-fluorobenzylamine $\quad 68 \quad 96$

4 2,6-fluorobenzylamine $\quad 34 \quad 91$

$5 \quad$ Isopropyl $L$-alaninate $45 \quad 68^{*}$

$6 \quad$ Cyclohexane methylamine $\quad 86 \quad 93.5$

$\begin{array}{llll}7 & \text { Cyclopropylamine } & 91 & 92.5\end{array}$

$8 \quad$ Cyclobutylamine 93.5

$\begin{array}{llll}9 & \text { Oleylamine } & 9 & 100\end{array}$

$10 \quad(S)$-1-phenylethan-1-amine $\quad 97 \quad 88.4$

$11 \quad(R)$-1-phenylethan-1-amine $\quad 97 \quad 97.5$
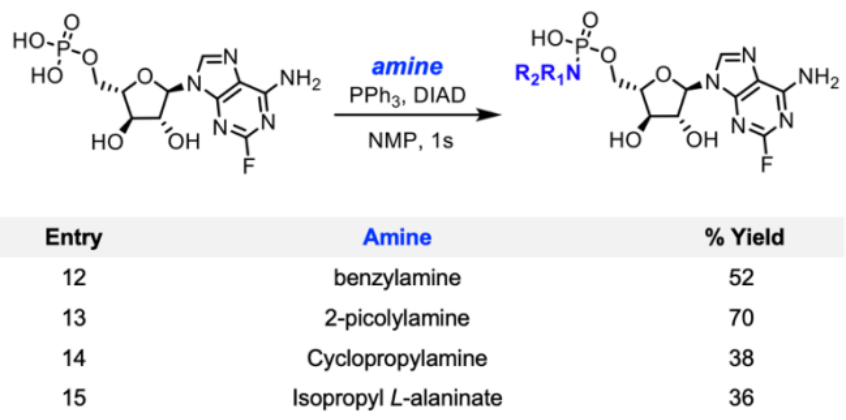

Figure 1. Structurally diverse amines can be efficiently attached under Mitsunobu conditions. (A) Reaction scope between a model phosphonic acid (BnHEX) and various amines. Yields were calculated via ${ }_{31} \mathrm{P}$ NMR spectroscopic analysis of the crude reaction mixture, using TPPO as an internal standard. (B) Reaction scope between model phosphoric and amines (1-naphtyl phosphate, top; Fludarabine monophosphate, bottom) and amines. For reactions described in $\mathbf{A}$ and $\mathbf{B}$ : Yield (1) was obtained without the addition of DBU, while yield (2) was obtained with the addition of 2 equivalents (A) or 0.75 equivalents (B) of DBU. (C) Highly functionalized molecules undergo selective amidation at $\mathrm{P}(\mathrm{V})$; reaction conditions were the same as for those described in $A$, with the addition of 2 equivalents of DBU. Yields are reported as the mean of 2 independent trials. For full synthetic procedures, see Supplementary Information. 


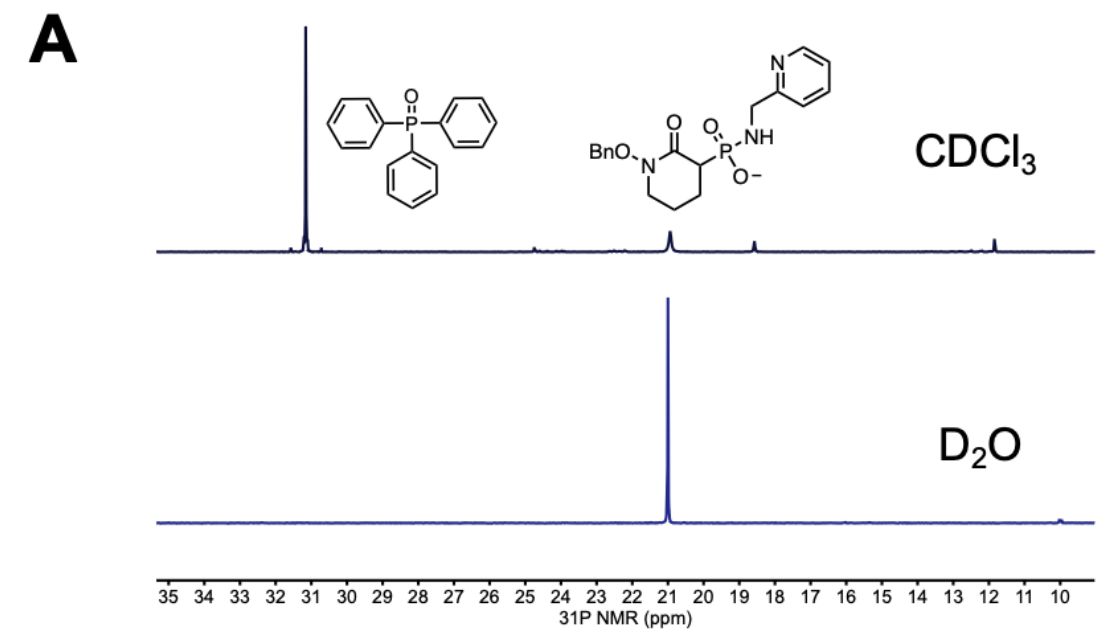

B

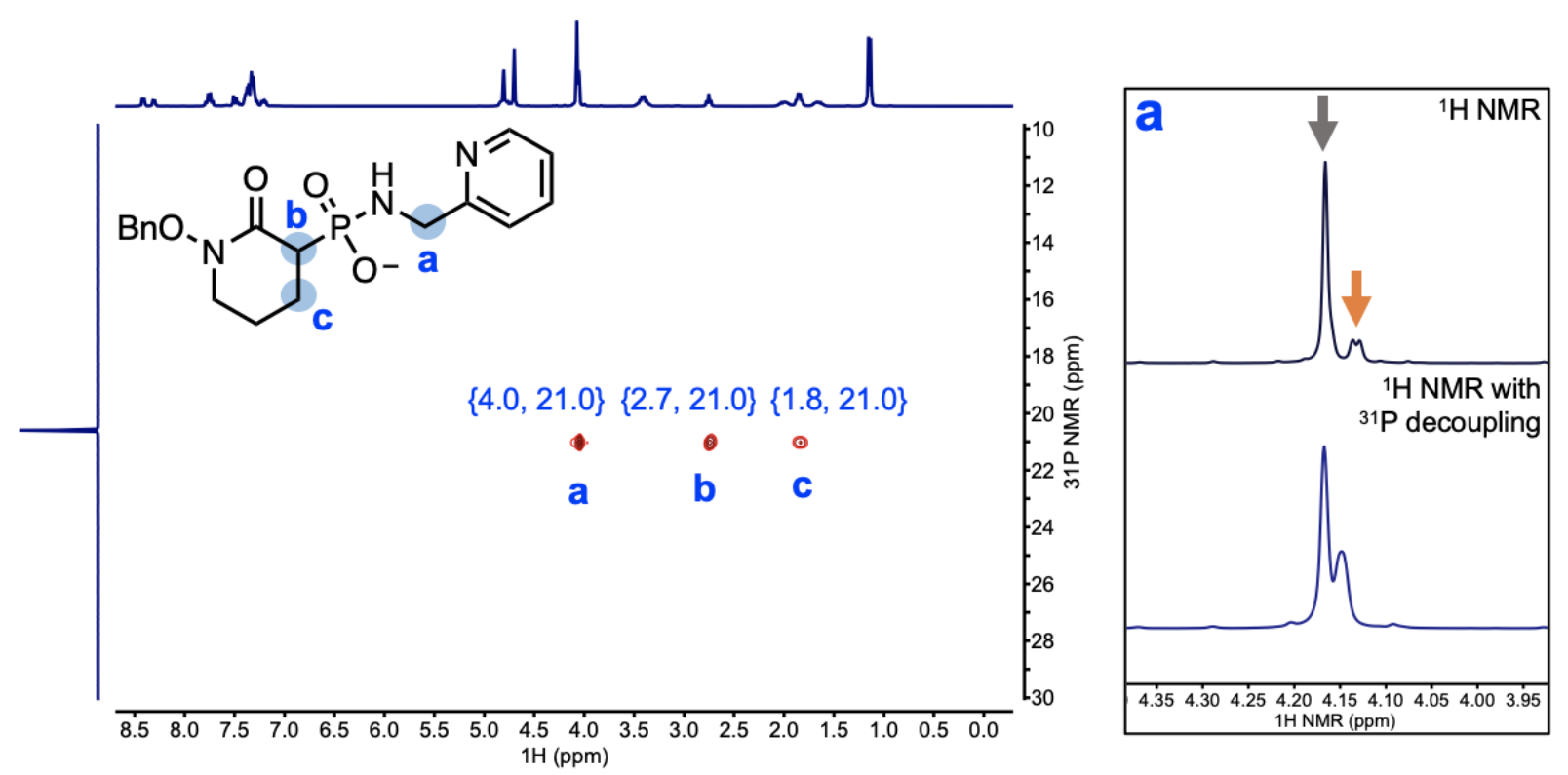

Figure 2. Negatively charged phosphoramidates and phosphonoamidates naturally lend themselves to a single water extraction for sufficiently clean product isolation. (A) ${ }_{31} P$ NMR spectra of the crude reaction mixture (top, $\mathrm{CDCl}_{3}$ ) and the aqueous solution after 1 water extraction (bottom, $\mathrm{D}_{2} \mathrm{O}$ ). Note the absence of TPPO ( 31 ppm) in the $\mathrm{D}_{2} \mathrm{O}$ extract. (B) Left: ${ }_{1} \mathrm{H}-{ }_{31} \mathrm{P}$ HSQC spectrum of BnHEX 2-picolylamine in $\mathrm{D}_{2} \mathrm{O}$. Right: ${ }_{1} \mathrm{H}$ NMR (top) and ${ }_{1} \mathrm{H}$ NMR with ${ }_{31} \mathrm{P}$ decoupling (bottom) spectra of the benzylic proton region for the 2-picolylamine adduct to BnHEX. Compared to the free amine (grey arrow), the benzylic protons of the adduct (orange arrow) are J3-coupled to the phosphorous of BnHEX, as indicated by the convergence of the doublet in the non-decoupled spectrum to a singlet in the decoupled spectrum (bottom). 
A

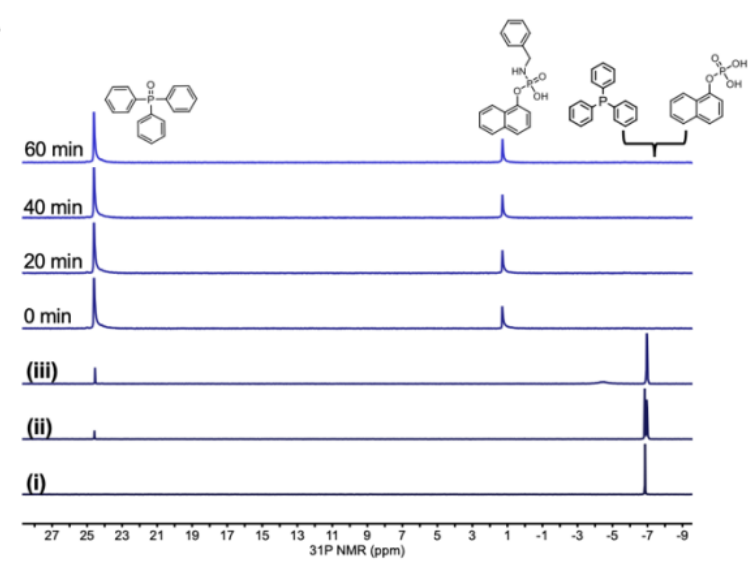

B
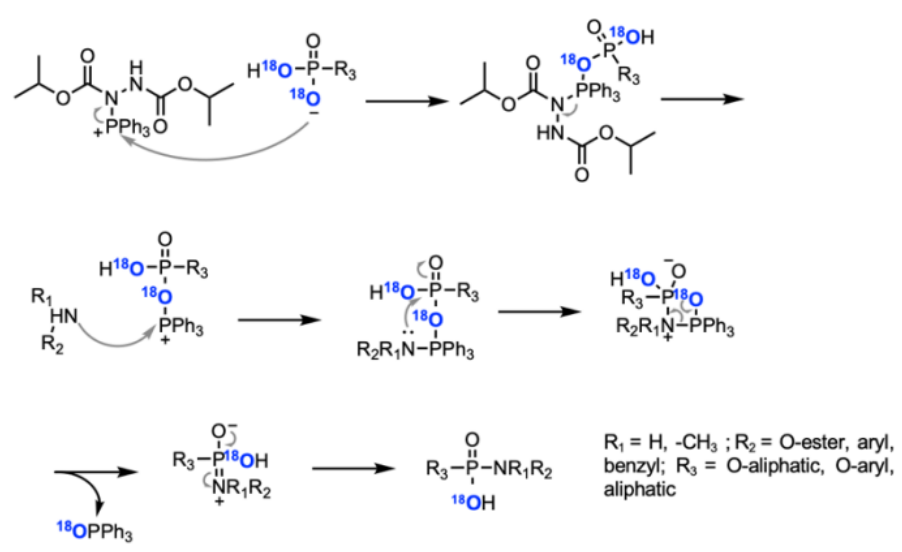

C
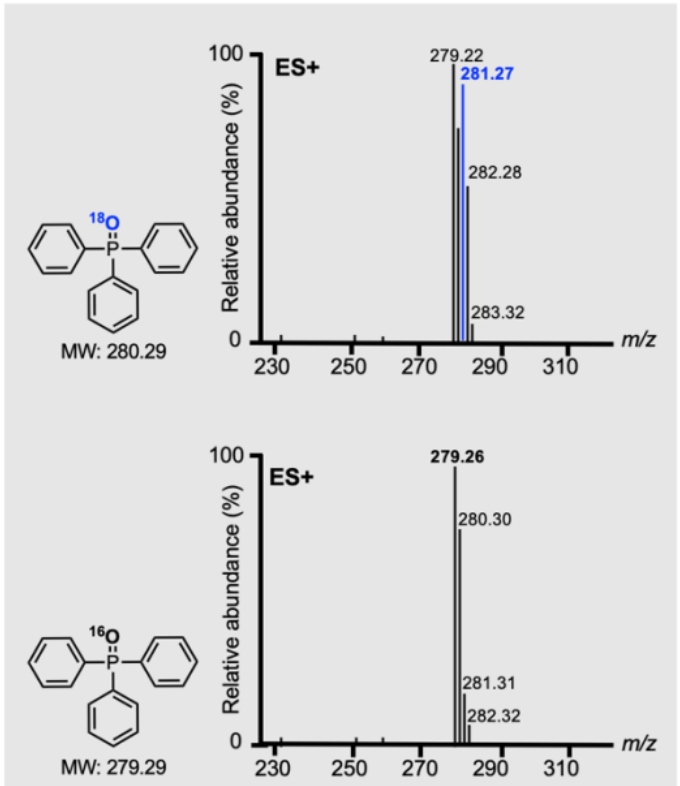
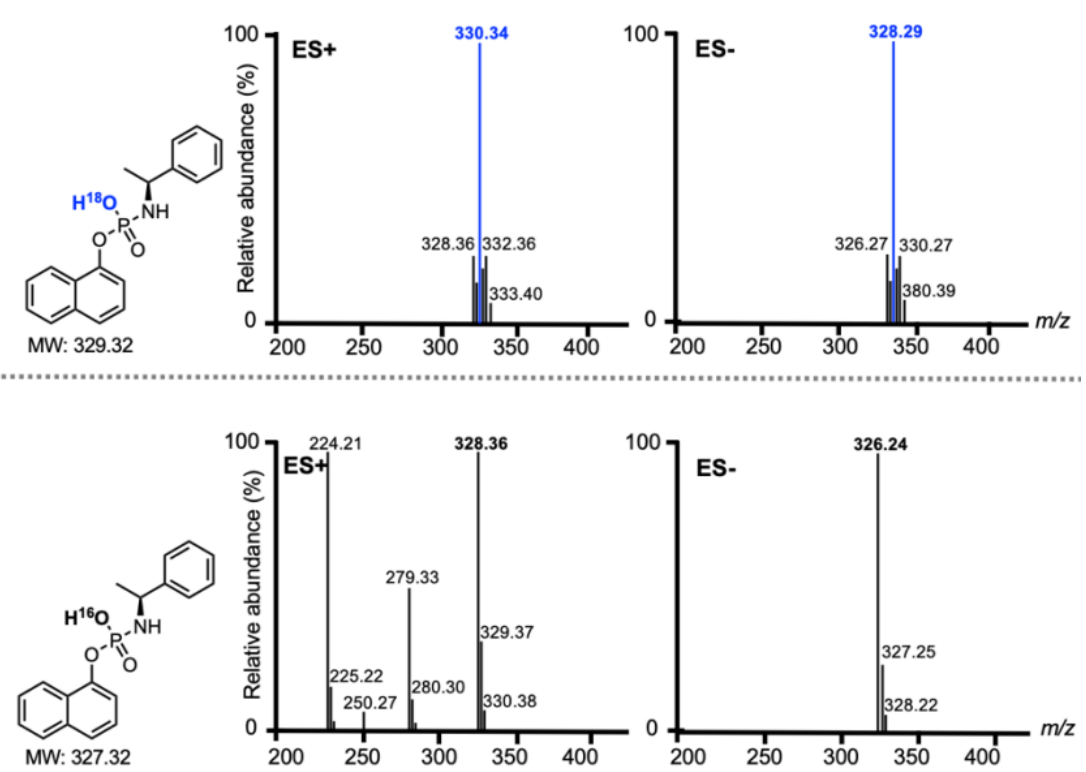

Figure 3. Mitsunobu coupling between amines and phosphates occurs instantaneously. (A) ${ }_{11} P$ NMR scans were taken at $121 \mathrm{MHz}$ in $80 \% \mathrm{NMP}, 20 \% \mathrm{CDCl}_{3}$. i. 1-naphthyl phosphate, ii. 1-naphthyl phosphate + $\mathrm{PPh}_{3}$, iii. 1-naphthyl phosphate $+\mathrm{PPh}_{3}+\mathrm{BnNH}_{2}$. Mitsunobu coupling between 1-naphthyl phosphate and benzylamine was monitored over 60 minutes. Chemical shifts are as follows (left to right, ppm): TPPO, +24.6; phosphoramidate product, +1.3; $\mathrm{PPh}_{3},-6.8$; 1-naphthyl phosphate, -6.9. (B) ${ }_{31} \mathrm{P}$ NMR spectra of the crude reaction mixture (top, $\mathrm{CDCl}_{3}$ ) and the aqueous solution after 1 water extraction (bottom, $\mathrm{D}_{2} \mathrm{O}$ ). Note the absence of TPPO ( 31 ppm) in the $\mathrm{D}_{2} \mathrm{O}$ extract. (C) Left: ${ }_{1} \mathrm{H}-31 \mathrm{P}$ HSQC spectrum of BnHEX 2-picolylamine in $\mathrm{D}_{2} \mathrm{O}$. Right: ${ }_{1} \mathrm{H}$ NMR (top) and ${ }_{1} \mathrm{H}$ NMR with ${ }_{31} \mathrm{P}$ decoupling (bottom) spectra of the benzylic proton region for the 2-picolylamine adduct to BnHEX. Compared to the free amine (grey arrow), the benzylic protons of the adduct (orange arrow) are J3-coupled to the phosphorous of BnHEX, as indicated by the convergence of the doublet in the non-decoupled spectrum to a singlet in the decoupled spectrum (bottom). 


\section{References}

[1] C. McGuigan, D. Cahard, H. M. Sheeka, E. De Clercq, J. Balzarini, J. Med. Chem. 1996, 39, 1748-1753.

[2] H. K. Bhatia, H. Singh, N. Grewal, N. K. Natt, J. Pharmacol. Pharmacother. 2014, $5,278$.

[3] C. Ballatore, C. McGuigan, E. De Clercq, J. Balzarini, Bioorg. Med. Chem. Lett. $2001,11,1053-6$.

[4] E. Lawitz, A. Mangia, D. Wyles, M. Rodriguez-Torres, T. Hassanein, S. C. Gordon, M. Schultz, M. N. Davis, Z. Kayali, K. R. Reddy, et al., N. Engl. J. Med. 2013, 368, 1878-1887.

[5] D. Siegel, H. C. Hui, E. Doerffler, M. O. Clarke, K. Chun, L. Zhang, S. Neville, E. Carra, W. Lew, B. Ross, et al., J. Med. Chem. 2017, 60, 1648-1661.

[6] U. Pradere, E. C. Garnier-Amblard, S. J. Coats, F. Amblard, R. F. Schinazi, Chem. Rev. 2014, 114, 9154-9218.

[7] V. C. Yan, K. L. Yang, E. S. Ballato, K. Arthur, D. K. Georgiou, F. L. Muller, ChemRxiv 2020, DOI 10.26434/CHEMRXIV.12033303.V1.

[8] Y.-H. Lin, N. Satani, N. Hammoudi, J. J. Ackroyd, S. Khadka, V. C. Yan, D. K. Georgiou, Y. Sun, R. Zielinski, T. Tran, et al., bioRxiv 2018, 331538.

[9] R. P. Volante, Tetrahedron Lett. 1981, 22, 3119-3122.

[10] D. L. McMinn, M. M. Greenberg, Tetrahedron Lett. 1997, 38, 3123-3126.

[11] D. L. Hughes, R. A. Reamer, J. J. Bergan, E. J. J. Grabowski, J. Am. Chem. Soc. $1988,110,6487-6491$.

[12] A. S. Alanazi, E. James, Y. Mehellou, ACS Med. Chem. Lett. 2019, 10, 2-5. 
[13] K. C. K. Swamy, N. N. B. Kumar, E. Balaraman, K. V. P. P. Kumar, Chem. Rev. 2009, 109, 2551-2651. 